\title{
Modeling and Identification of Passenger Car Dynamics Using Robotics Formalism
}

\author{
Gentiane Venture, Associate Member, IEEE, Pierre-Jean Ripert, Wisama Khalil, Senior Member, IEEE, \\ Maxime Gautier, and Philippe Bodson
}

\begin{abstract}
This paper deals with the problem of dynamic modeling and identification of passenger cars. It presents a new method that is based on robotics techniques for modeling and description of tree-structured multibody systems. This method enables us to systematically obtain the dynamic identification model, which is linear with respect to the dynamic parameters. The estimation of the parameters is carried out using a weighted least squares method. The identification is tested using vehicle dynamics simulation software used by the car manufacturer PSA Peugeot-Citroën in order to define a set of trajectories with good excitation properties and to determine the number of degrees of freedom of the model. The method has then been used to estimate the dynamic parameters of an experimental Peugeot 406, which is equipped with different position, velocity, and force sensors.
\end{abstract}

Index Terms-Identification, mobile robot dynamics, modeling, passenger car.

\section{INTRODUCTION}

C AR manufacturers have to design and build their cars faster than ever to meet the customers needs. Meanwhile, safety considerations are becoming more numerous and tests very strict. To fulfill these constraints, they have to make use of simulations and calculations as well as experiments. During the design of a car, simulation is used but not for the tuning of the prototype. In order to build tools that allow computing while tuning, it is necessary to have good knowledge of the prototype parameters for the different configurations of the car. Classical nonlinear identification techniques to estimate the dynamic parameters are very difficult to apply on the car model due to the complexity of its state space model. For instance, Schmitt et al. [1] use an output error method that is very time consuming because of the integration of the direct dynamic model (state space model) at each step of the optimization algorithm. Besides, this technique is very sensitive to nonlinear optimization issues such as initial values and local minima. We suggest identifying the dynamic parameters using

Manuscript received July 26, 2004; revised December 20, 2004, August 5, 2005, March 13, 2006, March 28, 2006, and April 13, 2006. This work was supported by PSA Peugeot-Citroën. The Associate Editor for this paper was B. De Schutter.

G. Venture is with Nakamura and Yamane Laboratory, School of Engineering, University of Tokyo, Tokyo 113-8656, Japan (e-mail: gentiane@ @nl.t. u-tokyo.ac.jp).

P.-J. Ripert and P. Bodson are with PSA Peugeot-Citroën, VelizyVillaboublay Cedex 78943, France (e-mail: pierrejean.ripert@mpsa.com; philippe.bodson@mpsa.com).

W. Khalil and M. Gautier are with the Institut de Recherche en Communication et Cybernétique de Nantes, Ecole Centrale de Nantes and Université de Nantes, Nantes Cedex 03 44321, France (e-mail: wisama.khalil@irccyn. ec-nantes.fr; Maxime.Gautier@irccyn.ec-nantes.fr).

Digital Object Identifier 10.1109/TITS.2006.880620 robotics techniques and tools that are based on an identification model that is linear with respect to the dynamic parameters. This dynamic identification model is obtained using the inverse dynamic model that can be obtained systematically using a recursive Newton-Euler algorithm [2]. The proposed method is tested by simulation and on an experimental Peugeot 406. The simulation is carried out using the dynamic vehicle simulation software ARHMM [3]. The influence of the car trajectory, the number of degrees of freedom (DOFs) of the model, as well as the cutoff frequency (cof) of filters on the results of the estimation is studied.

\section{VEHICLE DYNAMICS}

Vehicle dynamics is the study of vehicle behavior while driving. Only some elements of the vehicle are needed to describe and model this behavior; they constitute the DOFs of the car with respect to the ground and between the car components that link the chassis to the ground [4]. The car is composed of the following parts:

— the chassis;

— the steering system;

- the four suspensions and the two antiroll bars;

- the four unsprung bodies;

- the four wheels with their tires.

There are eight DOFs for each wheel [5], which are listed as follows:

- the track width (Fig. 1);

— the wheelbase (Fig. 1);

- the suspension clearance;

- the toe angle of the rear wheel and the steering angle of the front wheel;

— the camber angle (Fig. 1);

- the kingpin angle;

- the rotation of the wheel around its axis;

- the tire deflection.

Some of these DOFs are actuated, such as the steering angle or the rotation of the front wheels, but some others are elastic and elastokinematic deformations, such as suspension clearance. Car motion with respect to the ground is described by six DOFs (Fig. 2) - three translational and three rotational-which are listed as follows:

- longitudinal translation;

- lateral translation;

- vertical translation;

- roll: rotation around the longitudinal axis;

- pitch: rotation around the transversal axis;

- yaw: rotation around the vertical axis. 


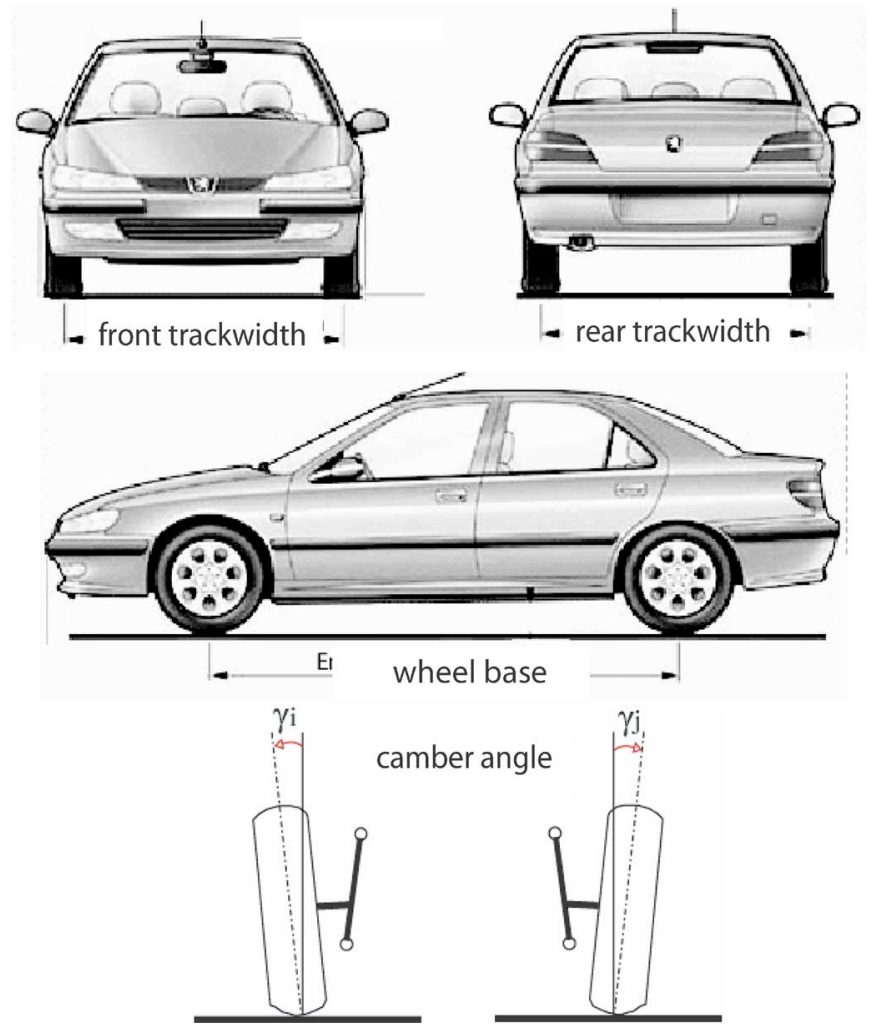

Fig. 1. Characteristic geometric parameters of the car dynamics. (Color version available online at http://ieeexplore.ieee.org.)

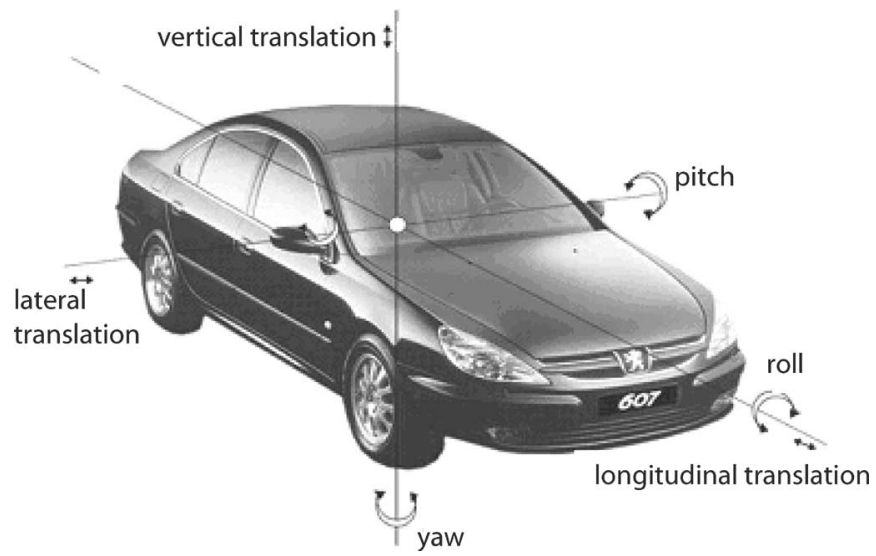

Fig. 2. Movements of the car body.

The external forces applied to the car, which have the most significant impact on vehicle dynamics, are the contact forces between the ground and the tires. These external forces are difficult to model [6], [7], or to estimate [8], [9], but they can be measured at the center of the wheels using dynamometric wheels. Aerodynamic forces also have an effect on the vehicle behavior, particularly at high speed $(>90 \mathrm{~km} / \mathrm{h})$ [4].

\section{Computation of the CAR Dynamics}

\section{A. Structure Description and Geometric Modeling}

To describe a passenger car, we will use the modified Denavit and Hartenberg (MDH) notations [2], [10] that are commonly used in robotics. This description allows us to obtain, sys-

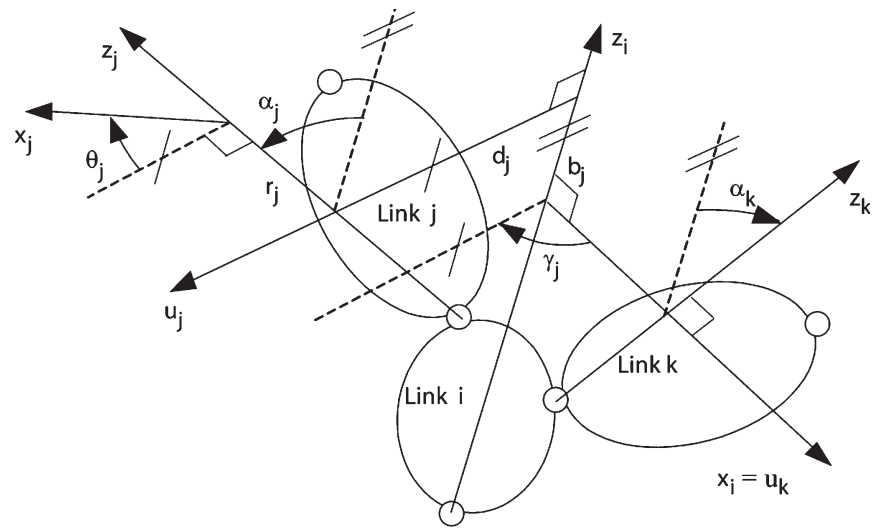

Fig. 3. Geometric parameters.

tematically, the identification dynamic model of the system, whatever the number of DOFs. The car is considered to be a tree-structured multibody system with $n$ bodies, where the wheels represent the terminal links. Each body $B_{j}$ is linked to its antecedent with a joint that represents an elementary DOF either translational or rotational, and the joint can be rigid or elastic. A body (or a link) can be real or virtual; the virtual bodies are introduced to describe joints with multiple DOFs or intermediate fixed frames. We define a reference frame $R_{i}$ $\left(O_{i}, \boldsymbol{x}_{i}, \boldsymbol{y}_{i}, \boldsymbol{z}_{i}\right)$ attached to each body $B_{i}$. The $\boldsymbol{z}_{i}$-axis is defined along the axis of joint $i$. The $\boldsymbol{u}_{j}$-axis is defined along the common normal between $\boldsymbol{z}_{i}$ and $\boldsymbol{z}_{j}$, where link $i$ is the antecedent of link $j$, which is denoted by $i=a(j)$. The $\boldsymbol{x}_{i}$-axis is defined arbitrarily along one of the axes $\boldsymbol{u}_{j}$, with $a(j)=i$. The $4 \times 4$ homogenous transformation matrix ${ }^{i} \boldsymbol{T}_{j}$ between two consecutive frames $R_{i}$ and $R_{j}$ is defined using the following six parameters [10] (Fig. 3):

- $\gamma_{j}$ : angle between $\boldsymbol{x}_{i}$ and $\boldsymbol{u}_{j}$ around the $\boldsymbol{z}_{i}$-axis;

- $b_{j}$ : distance between $\boldsymbol{x}_{i}$ and $\boldsymbol{u}_{j}$ along $\boldsymbol{z}_{i}$;

- $\alpha_{j}$ : angle between $\boldsymbol{z}_{i}$ and $\boldsymbol{z}_{j}$ around the $\boldsymbol{u}_{j}$-axis;

- $d_{j}$ : distance from $\boldsymbol{z}_{i}$ to $\boldsymbol{z}_{j}$ along $\boldsymbol{u}_{j}$;

- $\theta_{j}$ : angle between $\boldsymbol{u}_{j}$ and $\boldsymbol{x}_{j}$ around the $\boldsymbol{z}_{j}$-axis;

- $r_{j}$ : distance from $\boldsymbol{u}_{j}$ to $\boldsymbol{x}_{j}$ along $\boldsymbol{z}_{j}$.

In Fig. 3, since $\boldsymbol{x}_{i}$ is taken along $\boldsymbol{u}_{k}$, the parameters $\gamma_{k}$ and $b_{k}$ are equal to zero. The transformation matrix between frames $i$ and $j$ is represented by the following $4 \times 4$ matrix:

$$
{ }^{i} \boldsymbol{T}_{j}=\left[\begin{array}{cc}
{ }^{i} \boldsymbol{A}_{j} & { }^{i} \boldsymbol{P}_{j} \\
\mathbf{0}_{3 \times 1} & 0
\end{array}\right]
$$

where ${ }^{i} \boldsymbol{A}_{j}$ is the $3 \times 3$ rotation matrix of frame $j$ with respect to frame $i$, and ${ }^{i} \boldsymbol{P}_{j}$ is the $3 \times 1$ vector defining the origin of frame $j$ with respect to frame $i$.

The generalized coordinate of joint $j$ is denoted by $q_{j}$, and it is equal to $r_{j}$ if $j$ is translational and $\theta_{j}$ if $j$ is rotational. We define the parameter $\sigma_{j}=1$ if joint $j$ is translational and $\sigma_{j}=0$ if joint $j$ is rotational; $\overline{\sigma_{j}}=1-\sigma_{j}$. If there is no DOF between two frames that are fixed with respect to each other, we take $\sigma_{j}=2$. This means that the time derivative of $q_{j}$ is zero. 


\section{B. Dynamic Modeling}

1) Dynamic Parameters: A set of ten inertial parameters is associated with each real body $B_{j}$. It consists of

— the mass $M_{j}$;

- the six independent components of the inertia matrix $\boldsymbol{J}_{j}$ given in frame $R_{j}$, which are denoted by $X X_{j}, X Y_{j}$, $X Z_{j}, Y Y_{j}, Y Z_{j}$, and $Z Z_{j}$;

- the components $M X_{j}, M Y_{j}$, and $M Z_{j}$ of the first moments vectors $\boldsymbol{M} \boldsymbol{S}_{j}$ with respect to frame $R_{j}$.

When joint $j$ is elastic, we define the following parameters:

- the stiffness $k_{j}$ of the joint;

- the damping coefficient $h_{j}$;

— the Coulomb coefficient $f_{s j}$.

The vector of standard dynamic parameters of the system, which is denoted by $\boldsymbol{X}_{S}$, is composed of the previous parameters for all the links.

2) Lagrange Dynamic Model of the Car: The Lagrange formalism expresses the movement of each body in terms of the joint coordinates $\boldsymbol{q}=\left[q_{1}, \ldots, q_{n}\right]$, its first and second derivatives $\dot{\boldsymbol{q}}$ and $\ddot{\boldsymbol{q}}$, the external moments and forces applied on the system $\boldsymbol{F}_{e}$, and the vector of dynamic parameters $\boldsymbol{X}_{S}$. It is expressed as

$$
\boldsymbol{f}\left(\boldsymbol{q}, \dot{\boldsymbol{q}}, \ddot{\boldsymbol{q}}, \boldsymbol{F}_{e}, \boldsymbol{X}_{S}\right)=\mathbf{0} .
$$

To use the Lagrange method, the movement of the car body with respect to the ground is defined with a six-DOFs chain, where the first three are translational, and the last three are rotational [11], [12]. This chain is represented by five virtual bodies ( $B_{1}$ to $B_{5}$ ) with zero inertial parameters; the sixth body $B_{6}$ is the chassis. The reference body $B_{0}$ is the ground. In this case, the inverse dynamic model, which gives the joint torques, is obtained using the following general equation:

$$
\boldsymbol{\Gamma}+\boldsymbol{Q}=\boldsymbol{\Gamma}^{e}+\boldsymbol{\Gamma}^{f}+\boldsymbol{H}\left(\boldsymbol{q}, \dot{\boldsymbol{q}}, \ddot{\boldsymbol{q}}, \boldsymbol{X}_{S}\right)
$$

where $\boldsymbol{\Gamma}$ is the vector of joint forces or torques, and $Q$ is the vector of generalized efforts representing the projection of the external forces and torques on the joint axes. It is calculated using the following equation:

$$
\boldsymbol{Q}=-\sum \boldsymbol{G}_{j}(\boldsymbol{q})^{T} \boldsymbol{F}_{e j}
$$

where

$\boldsymbol{G}_{j}(\boldsymbol{q}) \quad$ Jacobian matrix of frame $R_{j}$

$\boldsymbol{F}_{e j} \quad$ vector of external forces $\boldsymbol{f}_{e j}$ and moments $\boldsymbol{m}_{e j}$ applied by body $B_{j}$ on the environment;

$\boldsymbol{H} \quad$ vector of inertial, Coriolis, centrifugal, and gravity forces;

$\Gamma^{e} \quad$ joint elastic force. The $j$ th element of $\boldsymbol{\Gamma}^{e}$ is written as follows:

If $j$ is an elastic joint, then

$$
\Gamma_{j}^{e}=k_{j} q_{j}+\text { off }_{j}
$$

with $q_{j}$ the joint coordinate $j$ with respect to the original position, $k_{j}$ the stiffness of joint $j$, and off $_{j}$ an offset.

if $j$ is not an elastic joint, then $\Gamma_{j}^{e}=0$;

$\Gamma^{f} \quad$ friction force. Friction is modeled using a viscous parameter $h_{j}$ and a Coulomb parameter $f_{s j}$ given as follows:

$$
\Gamma_{j}^{f}=h_{j} \dot{q}_{j}+f_{s j} \operatorname{sign}\left(\dot{q}_{j}\right) .
$$

In the following, we note that $\boldsymbol{L}=\boldsymbol{\Gamma}+\boldsymbol{Q}$.

3) Practical Calculation of the Lagrange Dynamic Model: The Lagrange model is typically calculated using the Lagrange equation, which calculates the kinetic and potential energies of all the elements of the mechanical system. The generalized forces $Q$ are calculated using (4) or by applying the virtual work principle [4], [7]. The Lagrange model can be calculated more easily using a recursive algorithm based on the NewtonEuler equation after expressing the link velocities and accelerations in terms of joint positions, velocities, and accelerations [10], [13]. This algorithm consists of two recursive calculations. The forward one calculates the total forces and moments on each body, whereas the backward one leads to calculation of the joint torques.

The forward recursive calculation can be summarized as follows: for $j=1$ to $n$, we calculate successively

$$
\begin{aligned}
{ }^{j} \boldsymbol{\omega}_{i}= & { }^{j} \boldsymbol{A}_{i}{ }^{i} \boldsymbol{\omega}_{i} \\
{ }^{j} \boldsymbol{\omega}_{j}= & { }^{j} \boldsymbol{\omega}_{i}+\bar{\sigma}_{j} \dot{q}_{j}{ }^{j} \boldsymbol{a}_{j} \\
{ }^{j} \dot{\boldsymbol{\omega}}_{j}= & { }^{j} \boldsymbol{A}_{i}{ }^{i} \dot{\boldsymbol{\omega}}_{i}+\bar{\sigma}_{j}\left(\ddot{q}_{j}{ }^{j} \boldsymbol{a}_{j}+{ }^{j} \boldsymbol{\omega}_{i} \times \dot{q_{j}}{ }^{j} \boldsymbol{a}_{j}\right) \\
{ }^{j} \dot{\boldsymbol{V}}_{j}= & { }^{j} \boldsymbol{A}_{i}\left[{ }^{i} \dot{\boldsymbol{V}}_{i}+\left({ }^{i} \widetilde{\boldsymbol{\omega}}_{i}+{ }^{i} \widetilde{\boldsymbol{\omega}}_{i}{ }^{i} \widetilde{\boldsymbol{\omega}}_{i}\right){ }^{i} \boldsymbol{P}_{j}\right] \\
& \quad+\sigma_{j}\left(\ddot{q}_{j}{ }^{j} \boldsymbol{a}_{j}+2{ }^{j} \boldsymbol{\omega}_{i} \times \dot{q}_{j}{ }^{j} \boldsymbol{a}_{j}\right) \\
{ }^{j} \boldsymbol{F}_{j}= & M_{j}{ }^{j} \dot{\boldsymbol{V}}_{j}+\left({ }^{j} \widetilde{\boldsymbol{\omega}}_{j}+{ }^{j} \widetilde{\boldsymbol{\omega}}_{j}{ }^{j} \widetilde{\boldsymbol{\omega}}_{j}\right){ }^{j} \boldsymbol{M} \boldsymbol{S}_{j} \\
{ }^{j} \boldsymbol{M}_{j}= & { }^{j} \boldsymbol{J}_{j}{ }^{j} \dot{\boldsymbol{\omega}}_{j}+{ }^{j} \boldsymbol{\omega}_{j} \times\left({ }^{j} \boldsymbol{J}_{j}{ }^{j} \boldsymbol{\omega}_{j}\right)+{ }^{j} \boldsymbol{M} \boldsymbol{S}_{j} \times{ }^{j} \dot{\boldsymbol{V}}_{j}
\end{aligned}
$$

where the upper left exponent denotes the projection frame, $x$ denotes the outer vector product $i=a(j)$, and

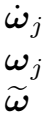

$\boldsymbol{\omega}_{j}$ angular acceleration of body $j$; angular velocity of body $j$; skew-symmetric matrix associated to the vector product, which is defined from the components of the $3 \times 1$ vector by the following equation:

$$
\widetilde{\boldsymbol{\omega}}=\left[\begin{array}{ccc}
0 & -\omega_{z} & \omega_{y} \\
\omega_{z} & 0 & -\omega_{x} \\
-\omega_{y} & \omega_{x} & 0
\end{array}\right]
$$

$\dot{\boldsymbol{V}}_{j}$

$\boldsymbol{F}_{j}$
$\boldsymbol{M}_{j}$ acceleration of $O_{j}$, which is the origin of frame $j$; total forces applied on body $j$; total moments applied on body $j$ with respect to $O_{j}$; 


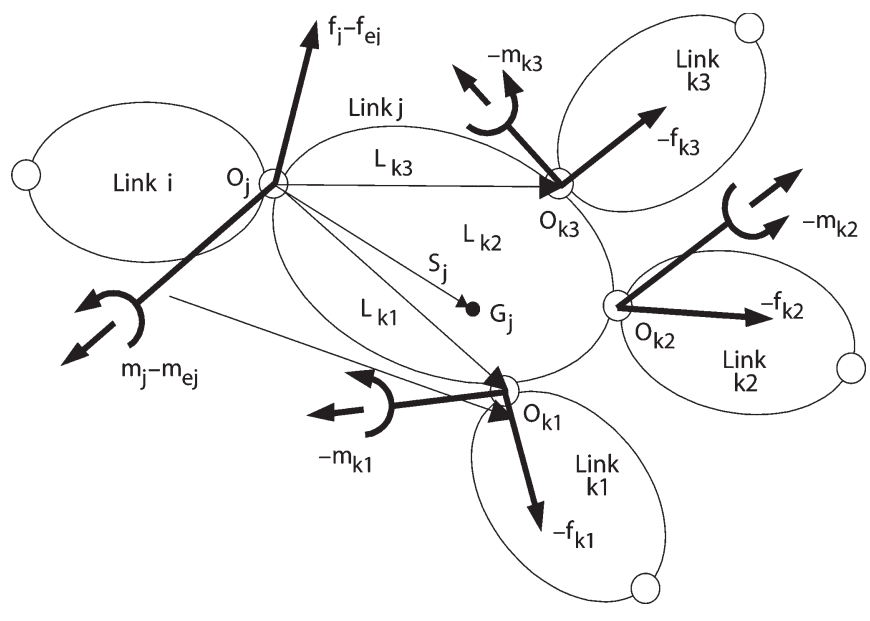

Fig. 4. Forces and moments acting on a link of a tree structure.

${ }^{j} \boldsymbol{A}_{i} \quad 3 \times 3$ orientation matrix of frame $R_{i}$ in $R_{j} ;$ $\boldsymbol{a}_{j} \quad$ unit vector along $\boldsymbol{z}_{j}$, thus, ${ }^{j} \boldsymbol{a}_{j}=\left[\begin{array}{lll}0 & 0 & 1\end{array}\right]^{T}$; $M_{j}, \boldsymbol{M} \boldsymbol{S}_{j}, \boldsymbol{J}_{j}$ defined in Section III-B-1.

The forward calculation is initialized with ${ }^{0} \boldsymbol{\omega}_{0}=\mathbf{0}$ and ${ }^{0} \dot{\boldsymbol{\omega}}_{0}=$ $\mathbf{0}$, whereas the translational acceleration of frame 0 will be set equal to gravity $\boldsymbol{g}$ with the opposite sign; thus, ${ }^{0} \dot{\boldsymbol{V}}_{0}=-\boldsymbol{g}$ in order to automatically take into account the effect of the gravity forces.

The backward recursive equations, for $j=n, \ldots, 1$ calculate the forces ${ }^{j} \boldsymbol{f}_{j}$ and moments ${ }^{j} \boldsymbol{m}_{j}$ exerted on body $B_{j}$ by its antecedent body $B_{i}$ (Fig. 4) as follows:

$$
\begin{aligned}
&{ }^{j} \boldsymbol{f}_{j}={ }^{j} \boldsymbol{F}_{j}+{ }^{j} \boldsymbol{f}_{e j}+\sum_{s(j)}{ }^{j} \boldsymbol{f}_{s(j)} \\
&{ }^{i} \boldsymbol{f}_{j}={ }^{i} \boldsymbol{A}_{j}{ }^{j} \boldsymbol{f}_{j} \\
&{ }^{j} \boldsymbol{m}_{j}={ }^{j} \boldsymbol{M}_{j}+{ }^{j} \boldsymbol{m}_{e j}+\sum_{s(j)}{ }^{j} \boldsymbol{A}_{s(j)}{ }^{s(j)} \boldsymbol{m}_{s(j)} \\
&+{ }^{j} \widetilde{\boldsymbol{P}}_{s(j)}{ }^{j} \boldsymbol{f}_{s(j)}
\end{aligned}
$$

where $s(j)$ indicates the bodies whose antecedent is body $B_{j}$, and ${ }^{j} \boldsymbol{f}_{e j},{ }^{j} \boldsymbol{m}_{e j}$ are the external forces and moments applied by body $B_{j}$ on the environment. The joint forces (or torques) are obtained by projecting ${ }^{i} \boldsymbol{f}_{j}$ (or ${ }^{i} \boldsymbol{m}_{j}$ ) on the joint axis $\boldsymbol{z}_{j}$ and by taking into account the effects of friction and elasticity as follows:

$$
\Gamma_{j}=\left(\sigma_{j}{ }^{j} \boldsymbol{f}_{j}+\bar{\sigma}_{j}{ }^{j} \boldsymbol{m}_{j}\right)^{T}{ }^{j} \boldsymbol{a}_{j}+\Gamma_{j}^{f}+\Gamma_{j}^{e} .
$$

This backward calculation is initialized by putting ${ }^{j} \boldsymbol{f}_{j}$ and ${ }^{j} \boldsymbol{m}_{j}$ equal to zero for the terminal links. We can note that the contact forces between the tire and the road will be taken into account through $\boldsymbol{f}_{e j}$ and $\boldsymbol{m}_{e j}$ of the terminal links (the wheels). The projection of these forces on the joint axes will be obtained systematically without application of (4), as would be the case if the Lagrange equation was used. It is to be noted that this algorithm can be programmed numerically or symbolically. To optimize the number of its operations, we use customized symbolic techniques to implement it [10]. It can be proven that the dynamic model is linear with respect to the standard dynamic parameters, thus, (3) can be rewritten as

$$
\boldsymbol{L}=\boldsymbol{D}_{S}(\boldsymbol{q}, \dot{\boldsymbol{q}}, \ddot{\boldsymbol{q}}) \boldsymbol{X}_{S}
$$

where matrix $\boldsymbol{D}_{S}$ is a function of $(\boldsymbol{q}, \dot{\boldsymbol{q}}, \ddot{\boldsymbol{q}})$.

The inertial parameters of the chassis appear explicitly in the first six equations giving $L_{1}$ to $L_{6}$.

4) Mixed Newton-Lagrange Model: The Newton-Euler formalism expresses the movement of a body in terms of its rotational speed, rotational acceleration, translational acceleration, and its current position $[\boldsymbol{\omega}, \dot{\boldsymbol{\omega}}, \dot{\boldsymbol{V}}, \boldsymbol{\Phi}]$.

We will suggest another form of the dynamic model that combines both Newton-Euler and Lagrange approaches in order to obtain a more efficient model of the car. In this case, the body of the car is represented by one body denoted by $B_{1}$ whose dynamic equations are expressed in terms of the Euler variables $\left[\dot{\boldsymbol{V}}_{1}, \boldsymbol{\omega}_{1}, \dot{\boldsymbol{\omega}}_{1}\right]$, whereas the rest of the system dynamics (i.e., bodies $B_{2}$ to $B_{n}$ ) is expressed in terms of Lagrange variables $(\boldsymbol{q}, \dot{\boldsymbol{q}}, \ddot{\boldsymbol{q}})$. The main advantages of this method are that the Euler variables of the chassis correspond to the measured variables in an experimental system and that the transformation matrices between the first six frames of the Lagrange model no longer exist. This enables us to obtain a more compact dynamic model and to reduce the number of mathematical operations required. The dynamic equations of this mixed model can be obtained by the recursive algorithm presented in Section III-B3 with the following modifications:

- The value of $n$ is reduced by 5 .

- In the forward recursive calculations, for $j=1$, the total forces and moments on the chassis $\boldsymbol{F}_{1}$ and $\boldsymbol{M}_{1}$ are calculated using (11) and (12) as a function of $\left[\dot{\boldsymbol{V}}_{1}, \boldsymbol{\omega}_{1}\right.$, $\dot{\omega}_{1}$ ]. For this first iteration, the other equations are not required.

- The equations of the chassis will be represented by the total forces and moments ${ }^{1} \boldsymbol{f}_{1}$ and ${ }^{1} \boldsymbol{m}_{1}$ exerted by link 0 on link 1 using (14)-(16); we note that (17) has no use in this case. Thus, the first six equations of the Lagrange model $\left(L_{1}, \ldots, L_{6}\right)$ will be replaced by the following six equations:

$$
\left[\mathbf{0}_{6 \times 1}\right]=\left[\begin{array}{c}
{ }^{1} \boldsymbol{f}_{1} \\
{ }^{1} \boldsymbol{m}_{1}
\end{array}\right] .
$$

${ }^{1} \boldsymbol{f}_{1}$ and ${ }^{1} \boldsymbol{m}_{1}$ are zero because there is no body antecedent to the chassis. The complete model can be expressed as a linear relation in the following dynamic parameters:

$$
\boldsymbol{L}=\boldsymbol{D}_{S}\left(\boldsymbol{\omega}_{1}, \dot{\boldsymbol{\omega}}_{1}, \dot{\boldsymbol{V}}_{1}, \boldsymbol{q}, \dot{\boldsymbol{q}}, \ddot{\boldsymbol{q}}\right) \boldsymbol{X}_{S} .
$$

5) Base Dynamic Parameters: The base dynamic parameters are the minimum number of parameters that can be used to compute the dynamic model, and they constitute the identifiable parameters that can be estimated using an identification method based on the dynamic model [10]. The base parameters are obtained from the standard dynamic parameters by grouping some parameters together and by eliminating those that have no effect on the dynamic model. Two methods are available for 
the computation of the base parameters, namely 1) a symbolical method [14] and 2) a numerical method based on the $Q R$ decomposition [15]. The numerical method allows considering the grouping relations due to the poor excitation properties of the chosen identification trajectory. After determining the identifiable parameters, the Lagrange dynamic identification model (18) becomes

$$
\boldsymbol{L}=\boldsymbol{D}(\boldsymbol{q}, \dot{\boldsymbol{q}}, \ddot{\boldsymbol{q}}) \boldsymbol{X}
$$

where $\boldsymbol{X}$ is the vector of $n_{B}$ base parameters, and $\boldsymbol{D}$ is the $n \times n_{B}$ matrix deduced from $\boldsymbol{D}_{S}$ by taking into account only the columns corresponding to the base parameters. A similar relation for the mixed Newton-Lagrange model can be deduced from (20).

\section{Identification Method}

We suggest making use of the fact that the dynamic model is linear in the dynamic parameters to identify the base parameters using linear least square optimization techniques [10], [16].

\section{A. Identification Model Sampling}

The dynamic model (21) is sampled along a trajectory. All the $n_{e}$ samples for the $n$ equations are collected in a linear system of equations as follows:

$$
\boldsymbol{Y}=\boldsymbol{W}(\boldsymbol{q}, \dot{\boldsymbol{q}}, \ddot{\boldsymbol{q}}) \boldsymbol{X}+\boldsymbol{\rho}
$$

where

$\boldsymbol{Y} \quad\left(n \times n_{e}\right) \times 1$ vector of joint torques obtained by sampling $\boldsymbol{L}$ and sorted by joint using the following equation:

$$
\boldsymbol{Y}=\left[\begin{array}{c}
\boldsymbol{Y}_{1} \\
\vdots \\
\boldsymbol{Y}_{n}
\end{array}\right]
$$

$\boldsymbol{Y}_{j} n_{e} \times 1$ vector of joint forces or torques associated with joint $j$;

$\boldsymbol{W} \quad\left(n \times n_{e}\right) \times n_{B} \quad$ observation matrix obtained by sampling $D$ and sorted by joint using the following equation:

$$
\boldsymbol{W}=\left[\begin{array}{c}
\boldsymbol{W}_{1} \\
\vdots \\
\boldsymbol{W}_{n}
\end{array}\right]
$$

$\boldsymbol{W}_{j} \quad n_{e} \times n_{B}$ observation matrix associated with joint $j$;

$\boldsymbol{\rho} \quad\left(n \times n_{e}\right) \times 1$ vector of modeling errors.

\section{B. Resolution and Interpretation of Results}

Equation (22) can be solved using the weighted least squares (WLS), which is implemented in many software packages with efficient algorithms (Matlab, Scilab). Because the equations are sorted by joint, the linear system is composed of $n$ subsystems, each with $n_{e}$ equations. The weighting procedure is defined in order to ensure the most significant equations [16], [17]. The weighted matrix $\boldsymbol{P}$ is computed using an estimation of the standard deviation for each joint subsystem $j \sigma_{\rho j}$ as follows:

$$
\boldsymbol{P}=\left[\begin{array}{lll}
\boldsymbol{S}_{1} & & \\
& \ddots & \\
& & \boldsymbol{S}_{n}
\end{array}\right]
$$

where

$\boldsymbol{S}_{j} \quad=\boldsymbol{I}_{n_{e}} / \sigma_{\rho j} ;$

$\boldsymbol{I}_{n_{e}} \quad\left(n_{e} \times n_{e}\right)$ identity matrix;

$\sigma_{\rho j} \quad$ calculated by the following equation:

$$
\sigma_{\rho j}^{2}=\frac{\left\|\boldsymbol{Y}_{j}-\boldsymbol{W}_{j} \hat{\boldsymbol{X}}^{j}\right\|^{2}}{n_{e}-n_{B j}}
$$

$n_{B j} \quad$ number of base parameters appearing in the equations of joint $j$;

$\hat{\boldsymbol{X}}^{j} \quad n_{B j} \times 1$ vector of estimated parameters using joint $j$ equations.

The weighted system to be solved is then given by

$$
\boldsymbol{Y}_{P}=\boldsymbol{W}_{P} \boldsymbol{X}+\boldsymbol{\rho}_{P}
$$

where $\boldsymbol{Y}_{P}=\boldsymbol{P} \boldsymbol{Y}, \boldsymbol{W}_{P}=\boldsymbol{P} \boldsymbol{W}$, and $\boldsymbol{\rho}_{P}=\boldsymbol{P} \boldsymbol{\rho}$.

Standard deviations on the estimated values $\sigma_{\hat{X} j}$ are computed using classical and simple results from statistics, considering the matrix $\boldsymbol{W}$ to be a deterministic one and $\rho$ to be a zero mean additive independent noise, with standard deviation such that

$$
\boldsymbol{C}_{\rho \rho}=E\left(\rho^{T} \rho\right)=\sigma_{\rho}^{2} \boldsymbol{I}_{n_{e} \times n}
$$

where $E$ is the expectation operator.

An unbiased estimation of $\sigma_{\rho}$ defined as follows is used:

$$
\sigma_{\rho}^{2}=\frac{\left\|\boldsymbol{Y}_{P}-\boldsymbol{W}_{P} \hat{\boldsymbol{X}}\right\|^{2}}{n \times n_{e}-n_{B}} .
$$

The covariance matrix of the estimation error and standard deviations can be calculated by

$$
\boldsymbol{C}_{\hat{X} \hat{X}}=E\left((\boldsymbol{X}-\hat{\boldsymbol{X}})(\boldsymbol{X}-\hat{\boldsymbol{X}})^{T}\right)=\sigma_{p}^{2}\left(\boldsymbol{W}_{P}^{T} \boldsymbol{W}_{P}\right)^{-1} .
$$

$\sigma_{\hat{X} j}=\left(\boldsymbol{C}_{\hat{X} \hat{X}}(j, j)\right)^{1 / 2}$ is the $i$ th diagonal coefficient of $\boldsymbol{C}_{\hat{X} \hat{X}}$. The relative standard deviation $\sigma_{\hat{X} j \%}$ is given by

$$
\sigma_{\hat{X} j \%}=100 \frac{\sigma_{\hat{X} j}}{\left|\hat{X}_{j}\right|} .
$$

Assuming that $\sigma_{\hat{X} j}$ is the realization of a Gaussian random variable, the $95 \%$ confidence interval is $2 \sigma_{\hat{X} j}$, and the relative confidence interval is $2 \sigma_{\hat{X} j} \%$. Then, we consider that a parameter with a relative confidence interval lower than $10 \%$ is well identified, keeping in mind that this is only an indicator based on statistical assumptions. The parameters that are not well identified may not be excited by the identification trajectory or may have only a small effect on the dynamic model so that they can be removed from the model [20]. However, it is to be noted that this criterion is not a deterministic one, particularly for 
parameters with small values, where they may be well identified although $\sigma_{\hat{X} j \%}$ is more than 10 .

\section{Filtering}

Some joint variables must be estimated by the differentiation or the integration of the measurements. Derivatives are estimated with digital zero-phase passband filters calculated as the product of a low-pass Butterworth filter and a central derivative algorithm (29). The Butterworth filter is implemented as a Matlab function filtfilt, which is a zero-phase forward and reverse digital filtering. Integrations are zero-phase-estimated as the product of a high-pass Butterworth filter and a trapezoidal algorithm (30) such that

$$
\begin{aligned}
f_{d}\left(t_{k}\right) & =\frac{f\left(t_{k+1}\right)-f\left(t_{k-1}\right)}{2 T_{e}} \\
f_{i}\left(t_{k}\right) & =f_{i}\left(t_{k-1}\right)+\frac{T_{e}}{2}\left(f\left(t_{k}\right)+f\left(t_{k-1}\right)\right)
\end{aligned}
$$

where

$$
\begin{array}{ll}
f_{d} & \text { derivative; } \\
f_{i} & \text { integral; } \\
T_{e} & \text { sampling period. }
\end{array}
$$

\section{Choosing a Trajectory With Sufficient Excitation}

The result of the estimation highly depends on the trajectory chosen for the identification [19], [20]. The excitation criteria are based on the calculation of a function of the condition number of the observation matrix $\boldsymbol{W}$ of the linear system [18]. Trajectories with sufficient excitation are defined using the simulation software ARHMM [3]. It has been shown that one kind of trajectory is enough to estimate the main dynamic parameters of a car, i.e., the "sinusoidal steering" at different speeds ranging from 90 to $160 \mathrm{~km} / \mathrm{h}$.

\section{EXPERIMENTAL CAR}

\section{A. Available Sensors}

Experiments are carried out on a Peugeot 406 car equipped with the following sensors:

- one SAGEM inertial unit giving the chassis angular velocities and translational accelerations with respect to the ground (cof: $10 \mathrm{~Hz}$ );

- four position sensors giving the clearance of the four suspension (cof: $20 \mathrm{~Hz}$ );

- one Correvit speed sensor giving lateral and longitudinal velocities of the chassis (cof: $15 \mathrm{~Hz}$ );

- four Zimmer laser sensors giving the four steering angles (cof: $10 \mathrm{~Hz}$ );

- four Eagle dynamometric wheels giving the four effort torques applied to the four wheel centers and the wheel angular position (cof: $100 \mathrm{~Hz}$ );

- four Zimmer laser sensors giving the vertical position of four points of the chassis with respect to the ground (cof: $20 \mathrm{~Hz}$ ).

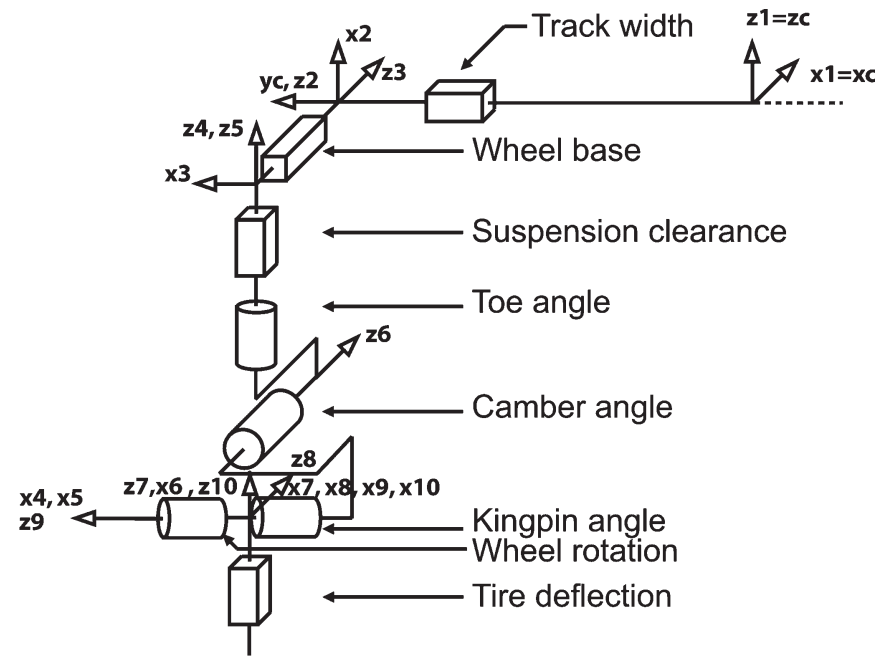

Fig. 5. Geometric modeling of the left rear branch (branch 3).

\section{B. Computation of the Missing Data}

Some joint variables required in the computation of the dynamic model cannot be measured by given sensors, particularly half track width, wheelbase, and kingpin variations. To access these variables, car manufacturers work with tabulation models. These are characterized on test benches and return geometric and elastokinematic deformations of the axle systems as a function of the suspension clearance and the position of the steering wheel. Half track width, wheelbase, and kingpin variations can be obtained in this way. The aerodynamic forces can also be obtained from tables as a function of the vehicle speed or must be identified (see Section V-D).

\section{Geometric Modeling}

The first modeling approach of the car dynamics is to consider the six DOFs between the ground and the chassis and all the DOFs between the chassis and the wheels. The corresponding system has 38 DOFs. The model is calculated using a mixed Newton-Lagrange, as presented in Section III-B4. The chassis dynamics is calculated using the Euler variables, whereas the branches are modeled using the Lagrange variables. This approach is more convenient with regard to the sensors available with our car, particularly with the use of an inertial unit. This model limits the projections of the measured variables on the car axes; thus, measurement noises are minimized. Moreover, there are about 30\% less mathematical operations (additions and multiplications) than with the Lagrange model.

For the left rear branch (Fig. 5), the DOFs are listed as follows:

- the half track width $l_{3}$;

- the wheelbase $L$;

- the suspension clearance $z_{3}$ (elastic joint);

- the toe angle $\beta_{3}$;

- the camber angle $\gamma_{3}$;

- the kingpin angle $\zeta_{3}$;

- the angular position of the wheel $\theta_{9}$;

- the vertical tire deflection $z_{t 3}$ (elastic joint). 
TABLE I

GeOMETRIC PARAMETERS OF THE LEFT REAR BRANCH (BRANCH 3) OF THE 38-DOF MODEL

\begin{tabular}{ccccccccc}
\hline \hline$j$ & $a(j)$ & $\sigma_{j}$ & $\gamma_{j}$ & $b_{j}$ & $\alpha_{j}$ & $d_{j}$ & $\theta_{j}$ & $r_{j}$ \\
\hline 1 & 0 & 2 & 0 & 0 & 0 & 0 & 0 & 0 \\
2 & 1 & 1 & 0 & 0 & $-\pi / 2$ & 0 & $-\pi / 2$ & $l_{3}$ \\
3 & 2 & 1 & 0 & 0 & $-\pi / 2$ & 0 & $-\pi / 2$ & $L$ \\
4 & 3 & 1 & 0 & 0 & $-\pi / 2$ & 0 & 0 & $z_{3}$ \\
5 & 4 & 0 & 0 & 0 & 0 & 0 & $\beta_{3}$ & 0 \\
6 & 5 & 0 & 0 & 0 & $\pi / 2$ & 0 & $\pi / 2+\gamma_{3}$ & 0 \\
7 & 6 & 0 & 0 & 0 & $\pi / 2$ & 0 & $\pi / 2+\zeta_{3}$ & 0 \\
8 & 7 & 2 & 0 & 0 & $\pi / 2$ & 0 & 0 & 0 \\
9 & 8 & 0 & 0 & 0 & $-\pi / 2$ & 0 & $\theta_{9}$ & 0 \\
10 & 8 & 1 & 0 & 0 & 0 & 0 & 0 & $z_{t 3}$ \\
\hline \hline
\end{tabular}

The geometric parameters of the left rear branch (branch 3) are presented in Table I. The chassis constitutes the first link, and its fixed frame $R_{1}$ is denoted by $R_{c}$. The fixed frame $R_{8}$ is used to define the dynamometer measuring frame.

The vertical tire deflection is represented by an elastic prismatic joint. The right rear branch (branch 4) is similar to the left rear branch but with $j=11$ to 19 . For the front, there is the same number of DOFs where the toe angle $\beta_{j}$ is replaced by the steering angle; thus, $j=20$ to 28 for the left front branch (branch 1) and $j=29$ to 37 for the right front branch (branch 2).

\section{Dynamic Identification Model}

The dynamic identification model (20) is obtained using the symbolic software package SYMORO+ [21], in which the algorithms given in Section III-B are implemented. Knowing the geometric parameters, this software automatically gives the identification model that is linear in the dynamic parameters.

Since the contact forces are measured at the center of the wheel by the dynamometric wheels, the inertial parameters of the wheels are not identified. Only chassis and unsprung body inertial parameters represent the inertial parameters to be identified. All the other bodies are virtual, and their inertial parameters are equal to 0 . The suspension parameters to be estimated are composed of stiffness and friction parameters and an offset as defined in (5) and (6). Antiroll bars are added at the front and rear to introduce a coupling between right and left suspension clearances. They are modeled by

$$
F_{\mathrm{ar} i}=k_{\mathrm{ar}}\left(q_{i}-q_{\mathrm{op} i}\right)
$$

$$
\begin{array}{cl}
\text { where } & \\
F_{\mathrm{ar} i} & \text { force due to the antiroll bar on suspension } i \\
k_{\mathrm{ar}} & \text { antiroll bar stiffness (in newtons per meter); } \\
q_{i} & \begin{array}{l}
\text { suspensions' clearance of the considered branch (in } \\
\text { meters); }
\end{array} \\
q_{\mathrm{op} i} & \begin{array}{l}
\text { suspensions' clearance of the opposite branch (in me- } \\
\text { ters). }
\end{array}
\end{array}
$$

Thus, two more parameters are needed, namely 1) $k_{\text {arr }}$ for the rear bar and 2) $k_{\text {arf }}$ for the front bar.
The aerodynamic forces and moments that are applied to the chassis are denoted by

$$
\begin{aligned}
{ }^{c} \tau_{a} & =\left\{\begin{array}{ll}
F_{x_{a}} & C_{x_{a}} \\
F_{y_{a}} & C_{y_{a}} \\
F_{z_{a}} & C_{z_{a}}
\end{array}\right\} \\
& =\left\{\begin{array}{cc}
\frac{1}{2} \rho_{\text {air }} S_{z} V_{a}^{2} & \frac{1}{2} \rho_{\text {air }} L S_{n} V_{a}^{2} \\
-\frac{1}{2} \rho_{\text {air }} S_{y} V_{a}^{2} & -\frac{1}{2} \rho_{\text {air }} L S_{m} V_{a}^{2}+z_{C} \frac{1}{2} \rho_{\text {air }} S_{x} V_{a}^{2} \\
-\frac{1}{2} \rho_{\text {air }} S_{x} V_{a}^{2} & -\frac{1}{2} \rho_{\text {air }} L S_{l} V_{a}^{2}+z_{C} \frac{1}{2} \rho_{\text {air }} S_{y} V_{a}^{2}
\end{array}\right\}
\end{aligned}
$$

where

${ }^{c} \tau_{a} \quad$ aerodynamic forces and torques given in the frame fixed to the chassis $R_{c}$ whose $\boldsymbol{x}_{c}, \boldsymbol{y}_{c}$, and $\boldsymbol{z}_{c}$ axes are along the longitudinal, lateral, and vertical axes (Fig. 2) of the chassis, respectively;

$\rho_{\text {air }} \quad$ density of the air;

$L \quad$ wheelbase;

$z_{C} \quad$ vertical position of the origin of the frame $R_{c}$ with respect to the front axle;

$S_{x}, S_{y}, S_{z}$ drag coefficients of the vehicle in the air along the longitudinal, transversal, and vertical directions;

$S_{l}, S_{m}, S_{n}$ drag coefficients of the vehicle in the air around the longitudinal, transversal, and vertical directions;

$V_{a} \quad$ norm of the vector $\boldsymbol{V}_{a}$ of aerodynamic velocity computed as $\boldsymbol{V}_{a}=\boldsymbol{V}_{\text {air }}-\boldsymbol{V}_{1}$;

$V_{\text {air }} \quad$ speed of the air given in $R_{c}$, and because it is not measured, it is supposed to be null;

$V_{1} \quad$ speed of the vehicle given in $R_{c}$.

These forces are identified via the following model:

$$
{ }^{c} \tau_{a}=\left\{\begin{array}{cc}
C_{x} V_{a}^{2} & C_{l} V_{a}^{2} \\
C_{y} V_{a}^{2} & C_{m} V_{a}^{2} \\
C_{z} V_{a}^{2} & C_{n} V_{a}^{2}
\end{array}\right\}
$$

where the $C_{i}$ parameters are added to the parameters to be identified.

\section{Simulation of the CAR Identification}

The car model is composed of 38 DOFs and five physical inertial bodies with ten inertial parameters for each. Furthermore, each of the suspensions needs four parameters, each of the antiroll bars and aerodynamic forces or torques also need one parameter. Thus, this model is quite complex and certainly needs to be simplified in order to be correlated with the accuracy of the measurements and the available trajectories. This section presents a study of the robustness of the dynamic model generated in the previous step with respect to trajectories and perturbations. The software package ARHMM [3] is used to generate trajectories without perturbation using a set of known parameters. This software uses a dynamic algorithm that represents the maximum phenomena that exist in a real 
car so that it can describe the whole range of uses of the car, and it is run with a real time core. It takes into account the driver's inputs through the transfer line and the steering wheel, as well as elastokinematic deformations, aerodynamics, and nonlinearity of the suspensions and antiroll bars. Contact forces with the ground are given by the Pacejka's magic formula [6], [7]. It gives a more precise model of a real car than the 38DOF model. The trajectory available is sinusoidal steering at different speeds. At first, the model with 38 DOFs is used to determine whether all the standard parameters are excited. Then, the model is modified consequently by deleting or grouping together some parameters.

\section{A. Aerodynamic Effect}

With the sinusoidal steering trajectory, it appears that aerodynamic contribution is constant. Indeed, it is proportional to the vehicle speed, which is constant in the tests used. Aerodynamic forces are therefore estimated from tabulations. Only the coefficient $C_{x}$ of the aerodynamic force along the $\boldsymbol{x}$-axis is identified because it is the most important component. The value of the other coefficients are too small to be identified.

\section{B. Robust Practical Model}

The following conclusions and simplifications have been deduced through different simulations using ARMMH and analyzing experimental data and results.

1) The results are sensitive to measurement cofs: To explain this sensitivity, a fast Fourier transform is applied to the measurements to determine the modes of each one. It appears that

- the data from the inertial unit are perturbed by the driveline (modes at 3.9-4.3-5.5 Hz);

- camber and steering angle measurements are perturbed by the driveline (modes at 3.9-4.3-5.5 Hz);

- only suspension clearances have "rebound of the wheel" mode information (mode at $12.7 \mathrm{~Hz}$ ).

To remove driveline perturbation, all the measurements are filtered with a low-pass filter having a cof of $3.125 \mathrm{~Hz}$ except the suspension clearance, which is not filtered to preserve the "rebound of the wheel" mode. The $3.125-\mathrm{Hz}$ value is chosen because it preserves mechanical modes of the chassis, which are between 0.4 and $3 \mathrm{~Hz}$.

2) Most of the unsprung body inertial parameters are not excited. Only their masses are to be considered, the other nine inertial parameters are removed from the identification.

3) Moreover, the track width, the wheelbase, the steering, the camber, or the kingpin has negligible influence in dynamic identification and can be removed from the model. This will be shown by applying the experimental tests to the complete model of 38 DOFs and a reduced model of 20 DOFs.

4) The dynamic equations to be used for the identification are restricted to the six components of the chassis and the four related to suspension clearances.
TABLE II

RESUlts With THE 38-DOF MODEL

\begin{tabular}{|c|c|c|c|c|c|}
\hline parameters & units & a priori & estimated & $\sigma_{\hat{X} j}$ & $\sigma_{\hat{X} j \%}$ \\
\hline$\overline{M_{C}}$ & $K g$ & 1508.5 & 1496.6 & 2.2 & 0.1 \\
\hline$M X_{c}$ & $m . \breve{K g}$ & 2421.1 & 2403.0 & 4.6 & 0.1 \\
\hline$M Y_{c}$ & $m . K g$ & 0 & 8.7 & 1.6 & 19.1 \\
\hline$M Z_{c}$ & $m \cdot K g$ & -15.01 & -12.2 & 3.7 & 30.5 \\
\hline$X X_{c}$ & $K g \cdot m^{2}$ & 622.15 & 513.8 & 10.3 & 2.0 \\
\hline$Y Y_{c}$ & $K g \cdot m^{2}$ & 5898 & 4856.7 & 132.3 & 2.7 \\
\hline$Z Z_{c}$ & $K g \cdot m^{2}$ & 6199 & 6581.0 & 52.5 & 0.7 \\
\hline$X Z_{c}$ & $K g \cdot m^{2}$ & 44.12 & 137.0 & 11.8 & 8.6 \\
\hline$X Y_{c}$ & $K g \cdot m^{2}$ & 76 & -11.2 & 21.7 & 193.8 \\
\hline$Y Z_{C}$ & $K g \cdot m^{2}$ & -13.00 & -95.3 & 27.7 & 29.0 \\
\hline$M_{1}$ & $K g$ & 24 & 41.2 & 1.4 & 3.4 \\
\hline$M_{2}$ & $K g$ & 24 & 17.4 & 0.9 & 5.6 \\
\hline$M_{3}$ & $K g$ & 24 & 25.6 & 1.0 & 4.1 \\
\hline$M_{4}$ & $K g$ & 24 & 25.6 & 0.8 & 3.3 \\
\hline$k_{a r r}$ & $N / m$ & 19938 & 23639.0 & 450.5 & 1.9 \\
\hline$k_{\text {arf }}$ & $N / m$ & 19033 & 21820.5 & 344.1 & 1.5 \\
\hline$k_{1}$ & $N / m$ & 20600 & 26608.9 & 748.1 & 2.8 \\
\hline$f_{s 1}$ & $N$ & & 9.1 & 3.3 & 36.4 \\
\hline$h_{1}$ & $\mathrm{~N} / \mathrm{m} / \mathrm{s}$ & 3200 & 3528.4 & 96.7 & 2.7 \\
\hline off $f_{1}$ & $N$ & & 12383.2 & 226.5 & 1.8 \\
\hline$k_{2}$ & $N / m$ & 20600 & 26254.9 & 644.7 & 2.4 \\
\hline$f_{s 2}$ & $N$ & & 35.8 & 2.2 & 6.3 \\
\hline$h_{2}$ & $\mathrm{~N} / \mathrm{m} / \mathrm{s}$ & 3200 & 2981.1 & 70.2 & 2.3 \\
\hline of $f_{2}$ & $N$ & & 12266.8 & 194.9 & 1.5 \\
\hline$k_{3}$ & $N / m$ & 22000 & 26856.4 & 876.9 & 3.2 \\
\hline$f_{s 3}$ & $N$ & & 2.1 & 3.0 & 144.2 \\
\hline$h_{3}$ & $N / m / s$ & 3800 & 3832.5 & 106.2 & 2.7 \\
\hline of $f_{3}$ & $N$ & & 10603.7 & 252.2 & 2.3 \\
\hline$k_{4}$ & $N / m$ & 22000 & 31663.3 & 911.0 & 2.8 \\
\hline$f_{s 4}$ & $N$ & & 5.0 & 3.2 & 64.1 \\
\hline$h_{4}$ & $\mathrm{~N} / \mathrm{m} / \mathrm{s}$ & 3800 & 2599.8 & 107.0 & 4.1 \\
\hline of $f_{4}$ & $N$ & & 12303.8 & 262.4 & 2.1 \\
\hline$C x$ & & & 1.17158 & 0.00311 & 0.2 \\
\hline
\end{tabular}

\section{PRActical IDEntification Results}

The identification method has been applied to an experimental Peugeot 406 car. Only sinusoidal steering trajectories are available. The 38-DOF and the reduced 20-DOF model are tested. The a priori parameters of the car, which were provided by Peugeot-Citroën are shown in Table II. They do not take into account the driver nor the equipment so they give only an idea about their values. It is to be noted that no a priori values are provided by the manufacturer for the Coulomb parameters.

\section{A. Identification With a 38-DOF Model}

Results of the identification on the model having 38 DOFs are given in Table II. As pointed out in Section IV-B, the identification relative standard deviation $\sigma_{\hat{X} j \%}$ is used to give the following interpretation to the results: If $\sigma_{\hat{X} j \%}<10 \%$, the parameter is considered as well identified. Nevertheless, this interpretation is not an absolute criterion; it could be even wrong if the parameter value is low. Applied to the obtained results, the following parameters are considered as being well identified, and the identification values are close to the a priori values: $M_{c}, M X_{c}, X X_{c}, Y Y_{c}, Z Z_{c}, X Z_{c}, M_{1}, M_{2}, M_{3}$, $M_{4}, k_{\text {arr }}, k_{\text {arf }}, k_{1}, h_{1}$, off $1, k_{2}, f_{s 2}, h_{2}$, off $_{2}, k_{3}, h_{3}$, off $_{3}$, $k_{4}, h_{4}$, off 4 , and $C_{x}$. The following parameters appear not to be well identified: $M Y_{c}, M Z_{c}, X Y_{c}, Y Z_{c}, f_{s 1}, f_{s 3}$, and $f_{s 4}$. However, because these parameters have low values, it is difficult to conclude. For instance, compared to the a priori 


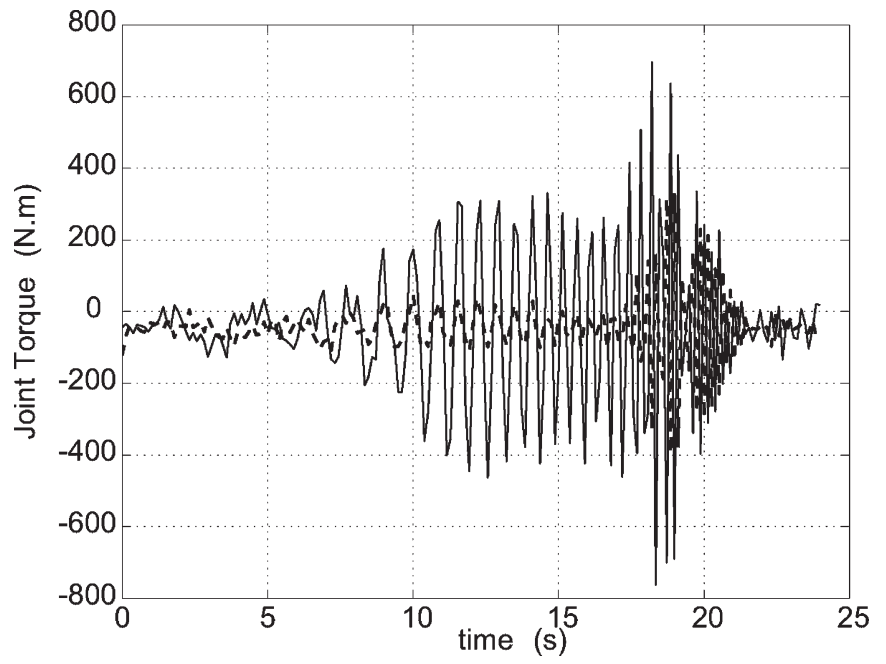

Fig. 6. Cross validation for the roll torque (in newton meters).

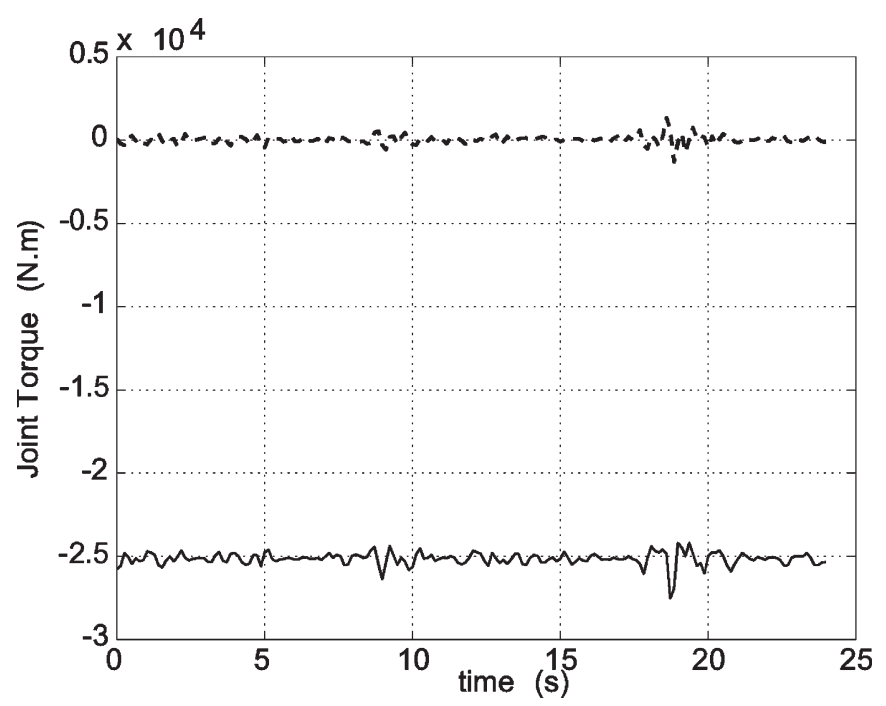

Fig. 7. Cross validation for the pitch torque (in newton meters).

values, $M Y_{c}$ and $M Z_{c}$ are good (taking into account that a variation of $10 \mathrm{~kg} \cdot \mathrm{m}$ on the value of a first moment component of the chassis is equivalent to a variation of $7 \mathrm{~mm}$ on the gravity center position since the chassis mass is about $1500 \mathrm{~kg}$ ). Parameters $X Y_{c}$ and $Y Z_{c}$ do not correspond to the a priori values, but because of their low value with respect to the diagonal elements $X X_{c}, Y Y_{c}$, and $Z Z_{c}$, they are physically hard to excite. Finally, concerning the parameters $f s_{1}, f s_{3}$, and $f s_{4}$, they have no a priori values, but their values are negligible with respect to the offset forces of the suspension, and thus, these parameters can be eliminated.

To validate the obtained values, the computed joint torques of the main joints, which are denoted by $\boldsymbol{Y}_{j}$, are compared with the reconstructed joint torques $\boldsymbol{W}_{j} \boldsymbol{X}$, where $\boldsymbol{X}$ is the vector of estimated parameters. Figs. 6-12 show cross validation results (the validation trajectory is a different sinusoidal steering trajectory than the one used for identification). Each figure shows the computed joint torque $\boldsymbol{Y}_{j}$, which is indicated by solid lines, and the corresponding error $\boldsymbol{W}_{j} \boldsymbol{X}-\boldsymbol{Y}_{j}$, which is indicated by broken lines. For suspension, only one branch is

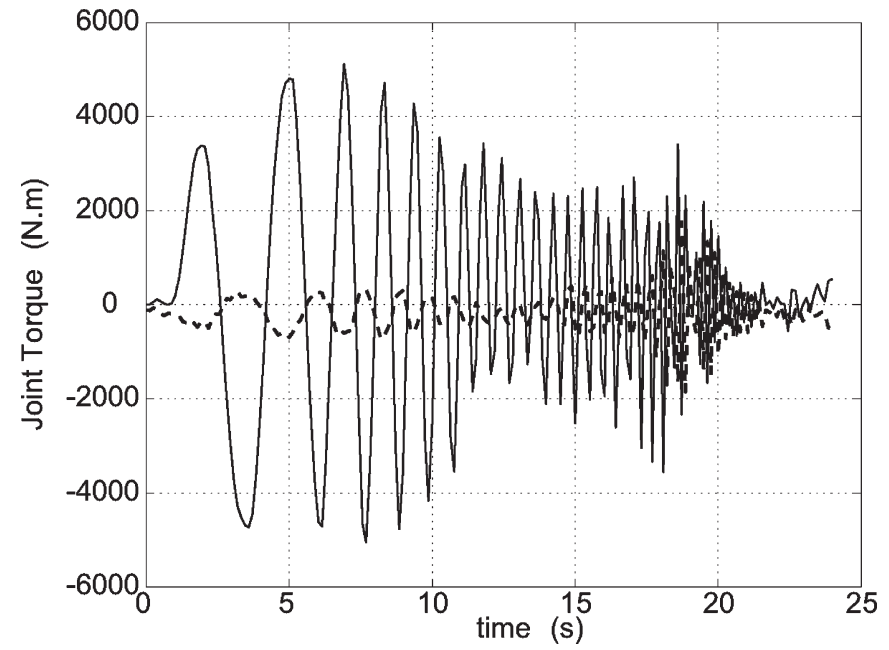

Fig. 8. Cross validation for the yaw torque (in newton meters).

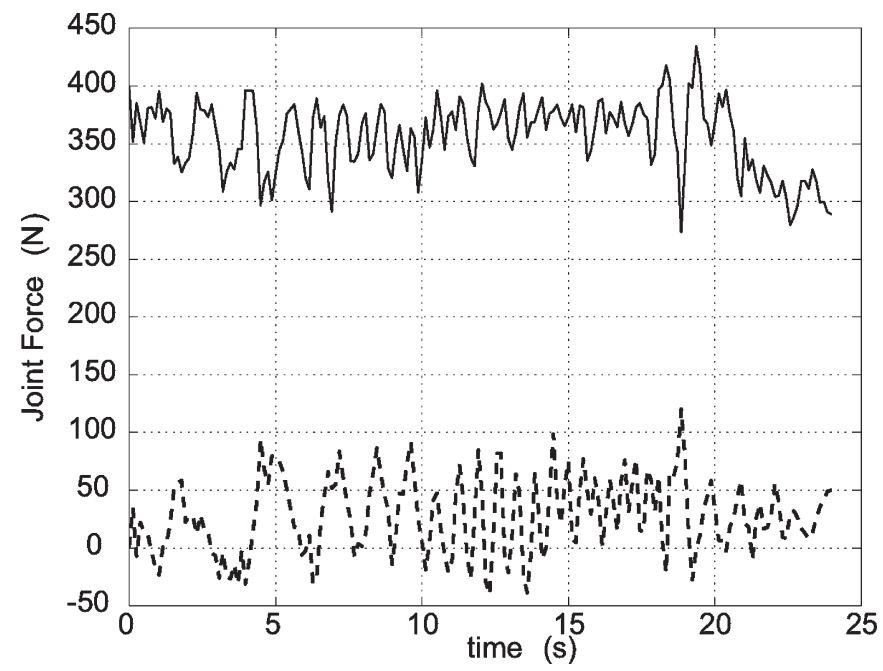

Fig. 9. Cross validation for the longitudinal force (in newtons).

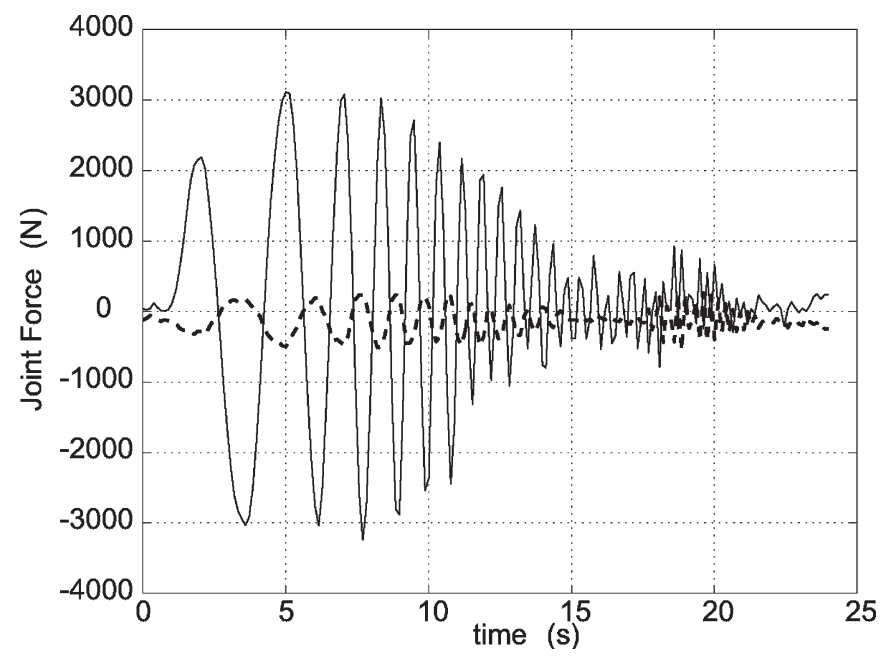

Fig. 10. Cross validation for the lateral force (in newtons).

given; the other branch results are similar. These curves show that the error signals are small with respect to the total force or moment signals. 


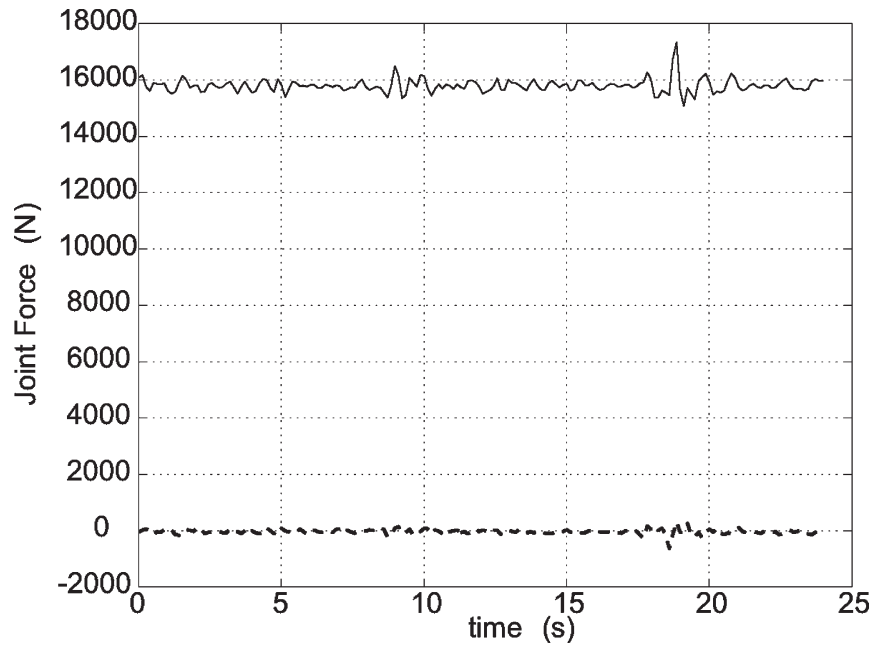

Fig. 11. Cross validation for the vertical force (in newtons).

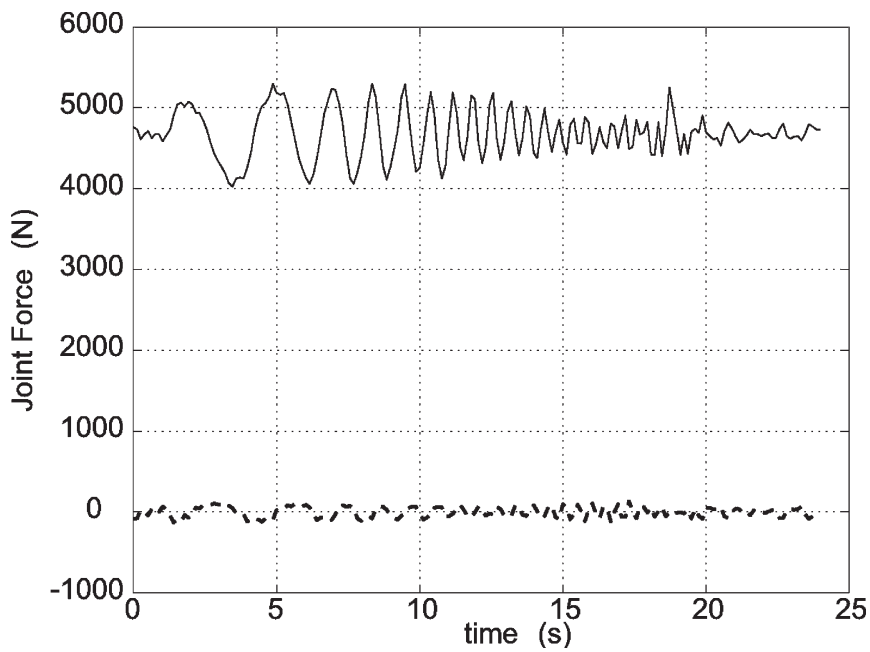

Fig. 12. Cross validation for the front left suspension force (in newtons).

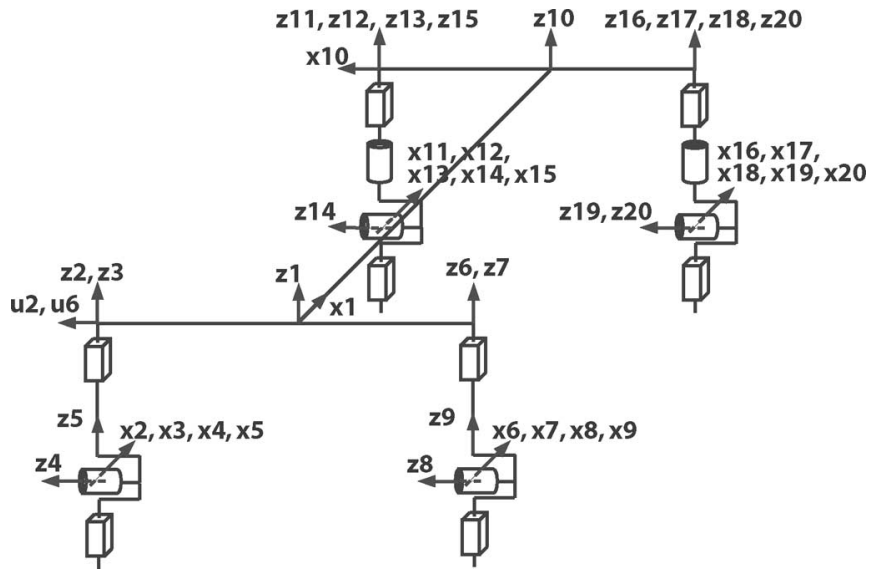

Fig. 13. 20-DOF model.

\section{B. Identification With a 20-DOF Model}

The 38-DOF model used for the previous identification is quite complex. Indeed, it takes into account half track width, wheelbase, toe, camber, and kingpin angle variations. The same identification procedure is applied using the reduced 20-DOF
TABLE III

Geometric Parameters of THE Reduced 20-DOF MOdel

\begin{tabular}{ccccccccc}
\hline$j$ & $a(j)$ & $\sigma_{j}$ & $\gamma_{j}$ & $b_{j}$ & $\alpha_{j}$ & $d_{j}$ & $\theta_{j}$ & $r_{j}$ \\
\hline 1 & 0 & 2 & 0 & 0 & 0 & 0 & 0 & 0 \\
2 & 1 & 1 & $\pi / 2$ & 0 & 0 & $l_{3}$ & $-\pi / 2$ & $z_{3}$ \\
3 & 2 & 2 & 0 & 0 & 0 & 0 & 0 & 0 \\
4 & 3 & 0 & 0 & 0 & $-\pi / 2$ & 0 & $\theta_{4}$ & 0 \\
5 & 3 & 1 & $\pi / 2$ & 0 & 0 & 0 & 0 & $z_{t 3}$ \\
6 & 1 & 1 & $\pi / 2$ & 0 & 0 & $-l_{4}$ & $-\pi / 2$ & $z_{4}$ \\
7 & 6 & 2 & 0 & 0 & 0 & 0 & 0 & 0 \\
8 & 7 & 0 & 0 & 0 & $-\pi / 2$ & 0 & $\theta_{8}$ & 0 \\
9 & 7 & 1 & 0 & 0 & 0 & 0 & 0 & $z_{t 4}$ \\
10 & 1 & 2 & 0 & 0 & 0 & $L$ & $\pi / 2$ & 0 \\
11 & 10 & 1 & 0 & 0 & 0 & $l_{1}$ & $-\pi / 2$ & $z_{1}$ \\
12 & 11 & 0 & 0 & 0 & 0 & 0 & $\beta_{1}$ & 0 \\
13 & 12 & 2 & 0 & 0 & 0 & 0 & 0 & 0 \\
14 & 13 & 0 & 0 & 0 & $-\pi / 2$ & 0 & $\theta_{14}$ & 0 \\
15 & 13 & 1 & 0 & 0 & 0 & 0 & 0 & $z_{t 1}$ \\
16 & 10 & 1 & 0 & 0 & 0 & $-l_{2}$ & $-\pi / 2$ & $z_{2}$ \\
17 & 16 & 0 & 0 & 0 & 0 & 0 & $\beta_{2}$ & 0 \\
18 & 17 & 2 & 0 & 0 & 0 & 0 & 0 & 0 \\
19 & 18 & 0 & 0 & 0 & $-\pi / 2$ & 0 & $\theta_{19}$ & 0 \\
20 & 18 & 1 & 0 & 0 & 0 & 0 & 0 & $z_{t 2}$ \\
\hline
\end{tabular}

TABLE IV

RESUlts WiTH THE 20-DOF MODEL

\begin{tabular}{|c|c|c|c|}
\hline parameters & estimated & $\sigma_{\hat{X} j}$ & $\sigma_{\hat{X} j \%}$ \\
\hline$M_{C}$ & 1497.1 & 2.2 & 0.1 \\
\hline$M X_{c}$ & 2401.6 & 4.6 & 0.1 \\
\hline$M Y_{c}$ & 8.2 & 1.6 & 20.0 \\
\hline$M Z_{C}$ & -17.3 & 3.7 & 21.5 \\
\hline$X X_{c}$ & 518.7 & 10.2 & 1.9 \\
\hline$Y Y_{c}$ & 4858.9 & 132.4 & 2.7 \\
\hline$Z Z_{c}$ & 6636.7 & 61.8 & 0.9 \\
\hline$X Z_{c}$ & 145.7 & 11.8 & 8.1 \\
\hline$X Y_{c}$ & -12.6 & 21.7 & 171.4 \\
\hline$Y Z_{c}$ & -95.9 & 27.7 & 28.9 \\
\hline$M_{1}$ & 41.0 & 1.3 & 3.3 \\
\hline$M_{2}$ & 17.3 & 0.9 & 5.6 \\
\hline$M_{3}$ & 25.0 & 1.0 & 4.1 \\
\hline$M_{4}$ & 25.8 & 0.8 & 3.2 \\
\hline$k_{a d r}$ & 23660.5 & 444.8 & 1.8 \\
\hline$k_{a d f}$ & 21800.0 & 339.6 & 1.5 \\
\hline$k_{1}$ & 26608.6 & 738.4 & 2.7 \\
\hline$f s_{1}$ & 9.1 & 3.2 & 35.9 \\
\hline$h_{1}$ & 3526.8 & 95.5 & 2.7 \\
\hline$o f f_{1}$ & 12384.3 & 223.6 & 1.8 \\
\hline$k_{2}$ & 26280.3 & 636.4 & 2.4 \\
\hline$f s_{2}$ & 35.9 & 2.2 & 6.2 \\
\hline$h_{2}$ & 2980.7 & 69.3 & 2.3 \\
\hline of $f_{2}$ & 12274.2 & 192.4 & 1.5 \\
\hline$k_{3}$ & 26815.8 & 865.7 & 3.2 \\
\hline$f s_{3}$ & 2.1 & 3.0 & 141.5 \\
\hline$h_{3}$ & 3832.4 & 104.8 & 2.7 \\
\hline$o f f_{3}$ & 10597.8 & 249.0 & 2.3 \\
\hline$k_{4}$ & 31628.4 & 899.5 & 2.8 \\
\hline$f s_{4}$ & 5.0 & 3.2 & 63.3 \\
\hline$h_{4}$ & 2597.5 & 105.7 & 4.0 \\
\hline$o f f_{4}$ & 12291.8 & 259.0 & 2.1 \\
\hline$C x$ & 1.1 & 0.00363 & 0.31192 \\
\hline
\end{tabular}

model shown in Fig. 13, whose geometric parameters are given in Table III.

Results of the identification on the 20-DOF model are given in Table IV. They are very similar to those given in Table II, which were obtained using the 38 -DOF model. Thus, we conclude that the 20-DOF model is sufficient for dynamic parameter identification. 


\section{CONCLUSION}

This paper presents a new method to estimate the dynamic parameters of a car. The identification method is based on the use of robotics formalism in modeling tree-structured multibody systems. The model presented takes into account the most important DOFs of the chassis with respect to the ground and the wheels (38 DOFs). The use of the Euler variables for the chassis and the Lagrange variables for the other elements of the car is more convenient with respect to the car sensors and reduces model complexity.

With the use of the vehicle dynamic software ARHMM and real tests, a reduced model with 20 DOFs has been deduced for the identification of the chassis, the unsprung bodies, and the suspension dynamic parameters. The sensors required are composed of an inertial unit, dynamometric wheels, and suspension clearance measurements. The aerodynamic coefficient force along the longitudinal axis has also been identified.

Future work will be focused on the model extension to the interaction between the wheels and the ground. This model extension would enable us to extend the identification to the contact forces model with the goal of avoiding the use of dynamometric wheels.

\section{REFERENCES}

[1] C. J. Schmitt et al., "Identification of physical parameters of a passenger car," in Proc. ECC, Karlsruhe, Germany, Sep. 1999, CD-ROM.

[2] W. Khalil and J.-F. Kleinfinger, "Minimum operations and minimum parameters of the dynamic model of tree structure robots," IEEE J. Robot. Autom., vol. RA-3, no. 6, pp. 517-526, Dec. 1987.

[3] P. Bodson, ARHMM V3.0 guide utilisateur, 2003. PSA—Peugeot Citroën, document interne edition.

[4] U. Kiencke and L. Nielsen, Automative Control Systems for Engine, Driveline and Vehicle. New York: Springer-Verlag, 2000.

[5] W. F. Milliken and D. L. Milliken, Race Car Vehicle Dynamics. Warrendale, PA: SAE International, 1995.

[6] H. B. Pacejka and I. J. M. Besselink, "Magic formula tyre model with transient properties," Veh. Syst. Dyn., vol. 127, no. 27, pp. 234-249, 1997.

[7] H. B. Pacejka, Tyre and Vehicle Dynamics. Oxford, U.K.: ButterworthHeinemann, 2002.

[8] C. Canudas de Wit, P. Tsiotras, E. Velenis, M. Basset, and G. L. Gissinger, "Dynamic friction models for road/tire longitudinal interaction," Veh. Syst. Dyn., vol. 39, no. 3, pp. 189-226, Mar. 2003.

[9] J. Stéphant, A. Charara, and D. Meizel, "Force model comparison on the wheel-ground contact for vehicle dynamics," in Proc. IEEE Intell. Veh. Symp., Versailles, France, 2002, pp. 100-104.

[10] W. Khalil and E. Dombre, Modeling, Identification and Control of Robots. London, U.K.: Hermès Penton, 2002.

[11] E. Guillo and M. Gautier, "Dynamic modeling and identification of earthmoving engines without kinematic constraints," in Proc. IEEE Int. Conf. Robot. and Autom., San Francisco, CA, Apr. 2000, pp. 2346-2351.

[12] G. Venture, W. Khalil, M. Gautier, and P. Bodson, "Dynamic modelling and identification of a car," in Proc. 15th IFAC World Congr., Barcelona, Spain, Jul. 2002, CD-ROM.

[13] J. Y. S. Luh, M. W. Walker, and R. C. P. Paul, "On-line computational scheme for mechanical manipulators," Trans. ASME, J. Dyn. Syst. Meas. Control, vol. 102, no. 2, pp. 69-76, Jun. 1980.

[14] W. Khalil and F. Bennis, "Symbolic calculation of the base inertial parameters of closed-loop robots," Int. J. Rob. Res., vol. 14, no. 2, pp. 112128, Apr. 1995.

[15] M. Gautier, "Numerical calculation of the base inertial parameters," J. Robot. Syst., vol. 8, no. 4, pp. 485-506, Aug. 1991.

[16] _ "Dynamic identification of robots with power model," in Proc. Int. Conf. Robot. Autom., Albuquerque, NM, 1997, pp. 1922-1927.

[17] G. Venture, W. Khalil, M. Gautier, and P. Bodson, "Parametric identification of a car dynamics," in Proc. IFAC Symp. Syst. Identif., Rotterdam, The Netherlands, Aug. 2003, pp. 295-300.

[18] C. Pressé and M. Gautier, "New criteria of exciting trajectories for robot identification," in Proc. IEEE Int. Conf. Robot. Autom., Atlanta, GA, 1993, pp. 907-912.
[19] M. Gautier and W. Khalil, "Exciting trajectories for inertial parameters identification," Int. J. Rob. Res., vol. 11, no. 4, pp. 362-375, 1992.

[20] M. Gautier, "Optimal motion planning for robot's inertial parameters identification," in Proc. 31st IEEE Conf. Decision Control, Tucson, AZ, 1992, vol. 1, pp. 70-73.

[21] W. Khalil and D. Creusot, "Symoro+: A system for the symbolic modelling of robots," Robotica, vol. 15, no. 2, pp. 153-161, Mar. 1997.

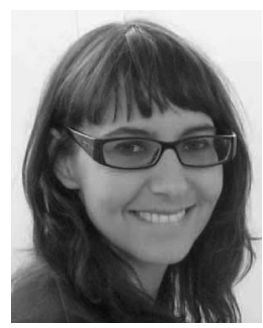

Gentiane Venture (M'05-A'05) received the engineering and the M.Sc. degrees in automatic control and robotics from the Ecole Centrale de Nantes, Nantes, France, in 2000, and the Ph.D. degree from the same university, in 2003, with a thesis on the dynamic modeling and identification of car dynamics, supported by PSA Peugeot-Citroën and the "Institut de Recherche en Communication et Cybernétique de Nantes" (IRCCyN), Nantes.

She is now a Research Fellow with the Nakamura and Yamane Laboratory, University of Tokyo, Tokyo, Japan, and is supported by the Japan Society for Promotion of Science. Her research interests are modeling, identification, and control of dynamic systems.

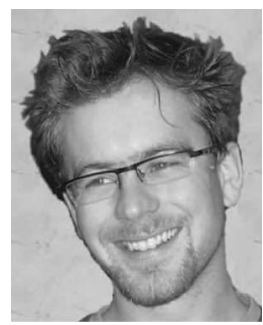

Pierre-Jean Ripert received the engineering degree and the M.Sc. degree in automatic control and robotics from the Ecole des Mines de Nantes and the Ecole Centrale de Nantes, Nantes, France, respectively, in 2003. He is currently working toward the Ph.D. degree, focusing on tire identification both from test benches and car measurements, and is supported by PSA Peugeot-Citroën and the "Institut de Recherche en Communication et Cybernétique de Nantes" (IRCCyN), Nantes.

His research interests are modeling, identification, interval analysis, and robust control

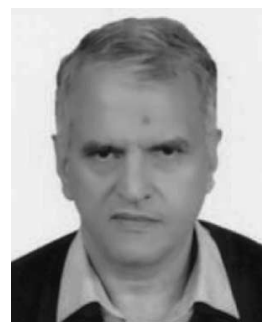

Wisama Khalil (SM'88) received the Ph.D. and "Doctorat d'Etat" degrees in robotics and control engineering from the University of Montpellier, Montpellier, France, in 1976 and 1978, respectively.

Since 1983, he has been a Professor with the Department of Automatic Control and Robotics, Ecole Centrale de Nantes, Nantes, France. He is carrying out his research with the Robotics Team, "Institut de Recherche en Communication et Cybernétique de Nantes" (IRCCyN). His research topics include modeling, control, and identification of robots.

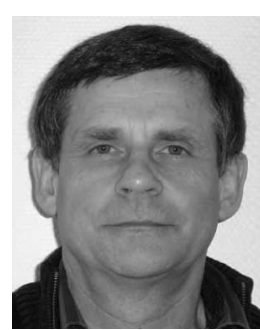

Maxime Gautier received the "Doctorat d'Etat" degree in robotics and control engineering from the University of Nantes, Nantes, France, in 1990.

Since 1991, he has been a Professor in automatic control with the Universite de Nantes. He is carrying out his research with the Robotics Team, "Institut de Recherche en Communication et Cybernétique de Nantes" (IRCCyN). His research topics include modeling, identification, and control of robots.

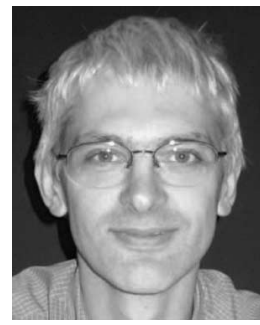

Philippe Bodson received the Diploma in electromechanical engineering from Liege University, Liege, Belgium, in 1991.

He was with SERA-CD, working in the field of vehicle dynamics until 1997. He is now with the French car manufacturer PSA Peugeot Citroën as Manager of the Vehicle Dynamics Modeling Team, with a specialty on the correlation between simulations and tests on track. 